\title{
Molecular analysis of keratocystic odontogenic tumor cell lines derived from sporadic and basal cell nevus syndrome patients
}

\author{
KAZUMA NOGUCHI $^{1 *}$, KEIKO WAKAI $^{1}$, TOHRU KIYONO ${ }^{2}$, MUTSUKI KAWABE $^{1}$, KYOHEI YOSHIKAWA $^{1}$, \\ TOMOKO HASHIMOTO-TAMAOKI ${ }^{3,4}$, HIROMITSU KISHIMOTO ${ }^{1}$ and YOSHIRO NAKANO ${ }^{3 *}$ \\ ${ }^{1}$ Department of Oral and Maxillofacial Surgery, Hyogo College of Medicine, Nishinomiya, Hyogo 663-8501; \\ ${ }^{2}$ Virology, Division of Carcinogenesis and Cancer Prevention, National Cancer Center Research Institute, Tokyo 104-0045; \\ ${ }^{3}$ Department of Genetics, Hyogo College of Medicine, Nishinomiya, Hyogo 663-8501; \\ ${ }^{4}$ Department of Clinical Genetics, Hyogo College of Medicine, Nishinomiya, Hyogo 663-8501, Japan
}

Received May 26, 2017; Accepted September 6, 2017

DOI: $10.3892 /$ ijo.2017.4146

\begin{abstract}
Keratocystic odontogenic tumor (KCOT) is a benign tumor often associated with basal cell nevus syndrome (BCNS). Mutations in Patched 1 (PTCHI), the Hedgehog (Hh) receptor, are responsible for BCNS. BCNS is distinguished by morphological anomalies and predisposition to benign and malignant tumors, including medulloblastoma, basal cell carcinoma, $\mathrm{KCOT}$ and ovarian fibromas. Among these tumors, KCOT is the least well studied because a suitable model system is not available for its investigation. To enable KCOT to be studied, we established two KCOT cell lines, one from a BCNS case (designated as iKCOT1) and one from a sporadic KCOT case (designated as sKCOT1). The BCNS-derived KCOT cell line, iKCOT1, retained a germline-mutated $P T C H 1$ allele and a wild-type $P T C H 1$ allele. The sporadic KCOT-derived KCOT cell line, sKCOT1, had different loss-of-function $P T C H 1$ mutations on both alleles. Both cell lines expressed stem cell markers (CD44, SOX2 and BMI1), mesenchymal cell markers (CDH2, VIM and SNAI2) and a neurogenic marker (NEFL). Culture of the cell lines in high calcium concentration media induced expression of epithelial cell and keratinocyte marker proteins (CDH1, CLDN1, KRT10 and IVL). Parakeratosis, which is characteristic for KCOTs, was observed in 2-D cultures. The similarities in protein expression patterns between the two cell lines suggested that common mechanisms underlie the
\end{abstract}

Correspondence to: Dr Kazuma Noguchi, Department of Oral and Maxillofacial Surgery, Hyogo College of Medicine, 1-1 Mukogawa-cho, Nishinomiya, Hyogo 663-8501, Japan

E-mail: knoguchi@hyo-med.ac.jp

Professor Yoshiro Nakano, Department of Genetics, Hyogo College of Medicine, 1-1 Mukogawa-cho, Nishinomiya, Hyogo 663-8501, Japan

E-mail: y-nakano@hyo-med.ac.jp

${ }^{*}$ Contributed equally

Key words: keratocystic odontogenic tumor, basal cell nevus syndrome, immortalization, 3-D culture development of both types of KCOT and a probable common origin of KCOT cells.

\section{Introduction}

Keratocystic odontogenic tumor (KCOT), previously known as odontogenic keratocyst, is a benign cystic lesion often manifesting locally in an aggressive fashion and recurring at a high rate (1). According to WHO classification, KCOT is defined as a benign neoplasm of odontogenic origin (2). Most KCOTs arise in the mandible, and KCOT is identified according to a characteristic histological appearance in which the cystic space is lined with a uniform parakeratinized squamous epithelium. The basal cells are aligned, with vertically elongated nuclei, and mitotic activity is higher than that of other odontogenic cysts (3). As described in detail previously (4), $5 \%$ of KCOTs occur as multiple lesions, and some of those cases occur as a manifestation of basal cell nevus syndrome (BCNS). BCNS, also known as Gorlin syndrome, is a rare autosomal dominant disorder (5), characterized by developmental abnormalities, such as calcification of the falx cerebri, multiple nevi, palmar and plantar pits, and skeletal deformity and predisposition to malignant cancers, including basal cell carcinoma (BCC), medulloblastoma, rhabdomyosarcoma, as well as benign tumors, such as KCOTs, ovarian and cardiac fibromas (6). Among these tumors, $>80 \%$ of BCNS patients from all populations suffer KCOTs. The gene encoding the Hedgehog $(\mathrm{Hh})$ receptor, Patched1 (PTCHI) has been identified to be responsible for BCNS (7). PTCH1 is a transmembrane co-receptor for Hh family proteins, which are secreted signaling molecules required for embryonic development and adult tissue homeostasis (8). Various types of PTCH1 mutations were found in most BCNS patients; however, a genotype-phenotype relationship was not observed $(9,10)$. After the discovery of the link between BCNS and Hh signaling, mutation analyses of sporadic BCNS-related neoplasms were intensively performed. Recent analysis showed that $>80 \%$ of sporadic KCOTs had a mutated PTCH1 gene (11). In addition, heterozygous Ptch1 knockout mice $\left(\right.$ Ptchl $\left.^{+-}\right)$, an animal model of BCNS (12), and $S u f u^{+-}$mice (13) also develop jaw keratocysts. Furthermore, overexpression of Gli2 under the control of the keratin 5 
promoter induced keratocyst formation (14). The animal model data strongly support the idea that activation of $\mathrm{Hh}$ signaling is an important factor in the induction of KCOTs. However, the study of KCOT has been hindered because a suitable in vitro biological system has not been available. To date, establishment of only one KCOT cell line has been reported (15). In this study, we successfully established immortalized KCOT cell lines from a patient with BCNS (iKCOT1) and from a sporadic case (sKCOT1) and found that these cells showed stem cell and mesenchymal cell properties. By exposing cells to a high calcium concentration, these cells showed epithelial keratinocyte properties. A possible mechanism of the onset of KCOTs will be discussed.

\section{Materials and methods}

Patients. KCOT tissues derived from BCNS patient NS11 or from a sporadic KCOT from a 25-year-old Japanese woman were kindly donated to our department. Patient NS11 was diagnosed as BCNS and a point mutation in intron 3 (c.584+2T $>\mathrm{G}$ ) of PTCHI was detected in this patient (10). The parents of the patients gave their written informed consent for participation in this study. This study was approved by the Medical Ethics Board of Hyogo College of Medicine (No. 108), and is in accordance with the principles expressed in the Declaration of Helsinki.

Cell culture and establishment of immortalized cells. KCOT cells (iKCOT1 and sKCOT1) were maintained in Epilife medium supplemented with HKGS (both from Cascade Biologics, Portland, OR, USA) or F-medium (16). Lentiviral vectors, CSII-CMV-cyclin D1, CSII-CMV-CDK4R24C and CSII-CMV-TERT were used to immortalize KCOT cells. Production and infection of recombinant lentiviruses with the vesicular stomatitis virus G glycoprotein (VSV-G) were described previously (17). The lentivirus backbone vector, CSII-MV-RfA, was a gift from Dr Miyoshi (Riken, BRC). After several passages, established cell lines were designated as iKCOT1 and SKCOT1.

Cell culture in 3-D life hydrogels. To analyze the ability to form 3-D structures, 3-D life hydrogel (Cellendes $\mathrm{GmbH}$, Reutlingen, Germany) was used. Cells were incubated in the Hydrogel in the presence of RGD-peptides for 12 days according to the manufacturer's MDCK cyst formation protocol. Cells were fixed in $4 \%$ formaldehyde in phosphatebuffered saline [PBS(-)]. After permeabilization, cells were stained with rhodamine phalloidin (Cytoskeleton, Denver, CO, USA) and mounted using Vectashield containing DAPI (Vector Laboratories, Burlingame, CA, USA). Imaging was carried out using a Zeiss LS780 confocal microscope.

DNA and RNA isolation and sequencing analysis. Genomic DNAs from KCOT cell lines were extracted using the DNeasy Blood and Tissue kit (Qiagen, Hilden, Germany). All coding exons and intron-exon boundaries were amplified by PCR and sequenced as described previously (10).

For analysis of mRNA, total RNA was extracted from a sKCOT1 cell line using the QIAamp RNA Blood mini kit (Qiagen) and subjected to RT-PCR using a Cells-to-cDNA ${ }^{\circledR}$ II kit (Applied Biosystems, Foster City, CA, USA) with random primers. The forward primer for exon 7 was 5'-GAATGGTG GATGTCATGGCTTATCC-3', and the reverse primer for exon 19 was 5'-ACGGCACTGAGCTTGATTCCGATGA-3'. Amplified products were purified and cloned into pCRII (Thermo Fisher Scientific, Waltham, MA, USA). Eleven randomly selected clones were sequenced using M13 forward or reverse primers.

Antibodies, immunostaining and western blotting. For immunofluorescence staining, cells were plated in 8-well culture slides (BD Bioscience, Bedford, MA, USA) and half of the medium in each well was changed to a high calcium medium $(1.23 \mathrm{mM}$; 1:1 mix of F medium and DMEM supplemented with 5\% FCS). Medium was changed every 3 days. Ten to twelve days later, cells were fixed in $4 \%$ paraformaldehyde in PBS(-) and permeabilized with $0.1 \%$ Tween-20 in PBS(-). After blocking in PBS(-) with $2 \%$ BSA, cells were subjected to immunofluorescence staining. Antibodies were visualized with Alexa-488 or Alexa-546 secondary antibodies (Thermo Fisher Scientific). Rhodamine phalloidin (Cytoskeleton) was used to detect actin filaments. DNA was stained with DAPI (Vector Laboratories). Imaging was carried out using a Zeiss LS780 confocal microscope.

For western blotting, iKCOT1 and sKCOT1 were harvested or incubated with low $(0.65 \mathrm{mM})$ or high $(1.23 \mathrm{mM})$ calcium medium for 10-12 days. Gel electrophoresis and western blotting were performed as described previously (18). Signals were detected by chemiluminescence using a Pierce SuperSignal western blotting kit (Thermo Fisher Scientific). All antibodies used are listed in Table I.

\section{Results}

Establishment of immortalized KCOT cell lines from patients with and without BCNS. We initially tried to culture cells from the KCOT of the patient with BCNS using standard culture methods for normal keratinocytes of the oral mucosa. In primary culture, KCOT cell colonies grew slowly, and predominantly consisted of closely packed polygonal cells with epithelial morphology. KCOT cells could be subcultured 3 to 4 times, but the polygonal cells were replaced by larger flattened cells and subsequently became senescent and could no longer be cultured. Therefore, we attempted to immortalize primary human KCOT cells by introducing CDK4R24C (inhibitor-resistant form of CDK4), cyclinD1 (which can inactivate $\mathrm{Rb}$ by phosphorylation) and hTERT genes using lentiviral vectors. The combination of CDK4R24C, cyclin D1 and hTERT resulted in efficient prolonged proliferation of the cells and they were designated as iKCOT1, and they were cultured for $>50$ passages. To our knowledge, this is the first study of the successful establishment of immortalized KCOT cells derived from a patient with BCNS. In addition, we also immortalized sporadic KCOT cells by the same method and designated this line as sKCOT1.

Identification of PTCH1 mutations in KCOT cell lines. We first analyzed the status of PTCHI in both cell lines. Sequence analysis of iKCOT1 cells showed a germline c.584+2T $>\mathrm{G}$ mutation (10) and a wild-type PTCHI sequence indicating no evidence of loss of heterozygosity $(\mathrm{LOH})$ at least in the coding region. RT-PCR analysis indicated that mutated and 
Table I. The antibodies used in this study.

\begin{tabular}{|c|c|c|}
\hline Name & Company & Cat no. \\
\hline Vimentin $(\mathrm{D} 21 \mathrm{H} 3) \mathrm{XP}^{\circledR}$, rabbit mAb & Cell Signaling Technology & 5741 \\
\hline E-Cadherin (24E10) rabbit mAb & Cell Signaling Technology & 3195 \\
\hline Slug (C19G7) rabbit mAb & Cell Signaling Technology & 9585 \\
\hline Claudin-1 antibody & Cell Signaling Technology & 4933 \\
\hline$\beta$-Catenin (D10A8) XP ${ }^{\circledR}$, rabbit mAb & Cell Signaling Technology & 8480 \\
\hline Anti-cytokeratin 10 antibody & Abcam & Ab76318 \\
\hline N-Cadherin (D4R1H) XP ${ }^{\circledR}$, Rabbit mAb & Cell Signaling Technology & 13116 \\
\hline Bmi1 (D20B7) XP ${ }^{\circledR}$, rabbit mAb & Cell Signaling Technology & 6964 \\
\hline Neurofilament-L (C28E10) Rabbit mAb & Cell Signaling Technology & 2837 \\
\hline Monoclonal anti-involucrin antibody produced in mouse & Sigma-Aldrich & I9018 \\
\hline Sox2 (D6D9) $\mathrm{XP}^{\circledR}$, rabbit mAb & Cell Signaling Technology & 3579 \\
\hline ZO-1 (D7D12) rabbit mAb & Cell Signaling Technology & 8193 \\
\hline CD44 (156-3C11) mouse mAb & Cell Signaling Technology & 3570 \\
\hline$\beta$-actin (D6A8) rabbit mAb & Cell Signaling Technology & 8457 \\
\hline Anti-mouse IgG (H+L chain) HRP & MBL & PM009-7 \\
\hline Anti-rabbit IgG (H+L chain) HRP & MBL & 458 \\
\hline Alexa Fluor 488 goat anti-mouse IgG (H+L) & Invitrogen & A11001 \\
\hline Alexa Fluor 546 goat anti-rabbit IgG $(\mathrm{H}+\mathrm{L})$ & Invitrogen & A11035 \\
\hline
\end{tabular}
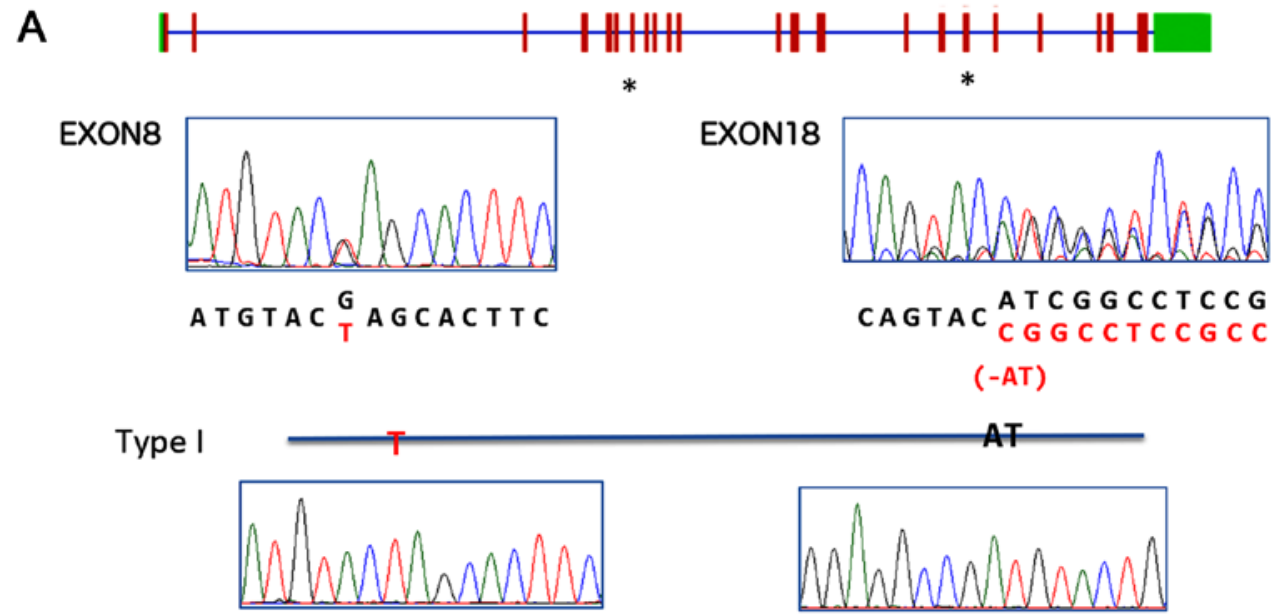

Type II
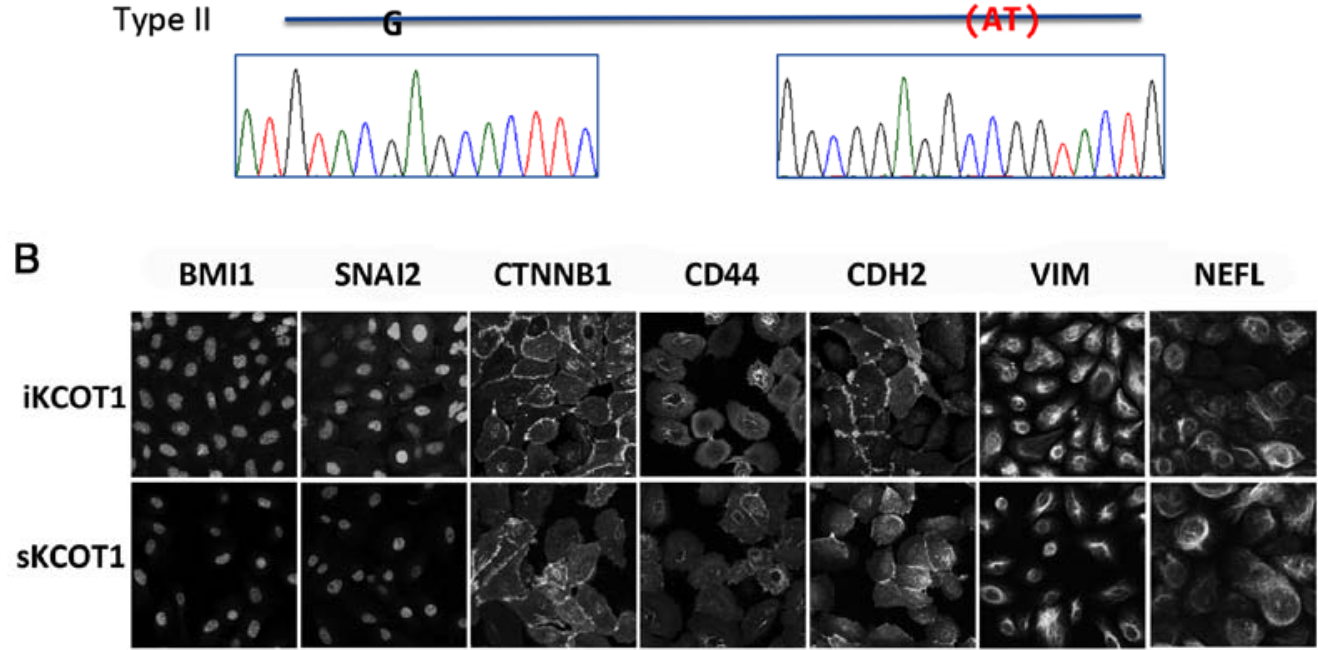

Figure 1. Characterization of the iKCOT1 and sKCOT1 cell lines. (A) PTCH1 mutations in sKCOT1 cell line. Upper, genomic analysis of PTCH1 in sKCOT1. Lower, cDNA analysis of PTCH1 in sKCOT1. (B) Immunostaining analysis of KCOT cells. BMI1, SNAI2, CTNNB1, CD44, CDH2, VIM and NEFL staining of cells grown in $\mathrm{F}$ medium at day 0 . 
wild-type alleles were equally expressed in this cell line (10). These data suggested that the wild-type allele is expressed in iKCOT1 cells. sKCOT1 cells showed two mutations in the PTCH1 gene: the c1120G $>$ T mutation in exon 8 is a nonsense mutation and the c3064_3065delAT mutation in exon 18 is a frameshift mutation (Fig. 1A). Both mutations were designated as loss of function mutations; therefore, we analyzed mRNA from sKCOT1 cells to address whether these mutations were on the same allele. After purification of mRNA from sKCOT1 cells, RT-PCR was performed using a primer set that covers exon 8 to 18 of the PTCH1 gene. Obtained fragments were cloned into pCRII-TOPO (Thermo Fisher Scientific) and 11 randomly selected independent clones were sequenced. Three out of 11 clones contained only the exon 8 c1120G $>$ T mutation and eight out of 11 clones had exon 18 c3064_3065delAT mutation only (Fig. 1A). These results indicated that each $\mathrm{PTCH} 1$ allele carried a single, different lethal mutation in sKCOT1 cells. Sequence analysis of the blood sample from the patient from which sKCOT1 cells were derived showed no mutations in the $P T C H 1$ gene indicating that these mutations were sporadic and that the patient was not BCNS. In addition, we sequenced the coding regions of the PTCH2, SMO and SUFU genes of both KCOT cells, but did not detect any mutation (data not shown).

Characterization in KCOT cell lines. To characterize the nature of the KCOT cell lines, immunostaining was performed. In both cell lines in F medium, the stem cell markers BMI1, SOX2 and CD44 and the mesenchymal cell markers SNAI2, $\mathrm{CDH} 2$ and VIM, were observed. Furthermore, both cell lines expressed NEFL, which is characteristic of neuronal cells (Fig. 1B).

Calcium treatment induced morphological changes and expression of epithelial or keratinocyte markers in KCOT cells. KCOT cells retain several characteristics of keratinocytes; therefore, we tested the response of the cell lines to calcium treatment by increasing calcium concentration of the culture media from 0.65 to $1.23 \mathrm{mM}$. Calcium-treated sKCOT1 cells stratified and parakeratinized, and iKCOT1 cells piled up and formed nodule-like structures by day 10 (Fig. 2A). Fig. 2B shows $\mathrm{CDH} 1$ and actin staining at 10 days of KCOT cells in low or high calcium medium. High expression of CDH1 was observed especially in nodule-like structure of iKCOT1 cells (Fig. 2B). When cells were cultured in dishes with high calcium medium for 12 days, sKCOT1 cells were stratified with the surface cells being of bigger cell size and with bigger nuclei compared with the bottom cells. Fig. 2C shows sequential images of CDH1 (green) and DAPI (magenta) stained sKCOT1 cells. The upper cells, predicted to be differentiated cells, expressed $\mathrm{CDH} 1$ and parakeratinized. Fig. 3 shows sequential images of sKCOT1 and iKCOT1 cells in 3-D hydrogels in low calcium concentration. Under low calcium conditions, sKCOT1 cells formed spheroids with nuclei at the center that were small and condensed, and lacked cell membranes (Fig. 3A). Conversely, iKCOT1 cells formed spheroids with normal nuclei and cell membranes (Fig. 3B). However, under high calcium conditions, some nuclei were condensed and lacked cell membranes, and were located at the center of the iKCOT1 spheroids similar to sKCOT1. These data indicated that both cell lines showed cyst-like structures in 3-D hydrogels with different calcium concentrations.

Because calcium treatment induced dramatic morphological changes to the KCOT cells, we examined protein levels by western blotting (Fig. 4). In both cell lines, CDH1, CLDN1, KRT10 and IVL were induced by calcium. However, levels of CDH2, VIM, TJP1, SNAI2, CD44 and BMI1 were reduced. CTNNB1 expression was reduced in sKCOT1 cells but was not changed in iKCOT1 cells. Although an increase of calcium concentration induced these changes in protein expression, the expression of some proteins changed with respect to time. Therefore, other factors, such as cell-to-cell contact or cell proliferation, may also be involved in changes in protein expression. NEFL expression was not influenced by calcium.

\section{Discussion}

Recent studies have demonstrated that mutations in $\mathrm{Hh}$ signaling components induce tumors in various types of cells. However, Hh signaling, especially during tumor formation, is not fully understood. BCNS is a very rare inherited disease caused by the misregulation of Hh signaling (19). Activation of Hh signaling, mainly due to the loss of PTCH1 function, causes benign and malignant tumors in BCNS patients. Therefore, study of BCNS derived tumors will provide clues to comprehend Hh-related tumors, especially tissue and ethnic specificity. Our group has intensively analyzed BCNS and its tumors $(10,20,21)$. Among BCNS-associated tumors, the study of KCOTs has been hindered because of the lack of a suitable model system. We therefore established sporadic and BCNSderived KCOT cell lines and analyzed their characteristics.

PTCH1 mutations and tumor formation. iKCOT1 from a BCNS patient and SKCOT1 from a non-BCNS sporadic patient have mutations in PTCH1. iKCOT1 cells have a germline mutation of c.584+2T>G (10) in one allele but we found no additional mutations on the other allele. RT-PCR analysis showed the existence of normal PTCH1 transcripts indicating that one of the PTCHI alleles appears to be wild-type.

Tumors occurring in Japanese BCNS patients are usually KCOTs, and BCCs are sometimes observed in older patients (10). BCC occurs at a low rate in African-American patients, but at a high rate (up to 97\%) in Caucasian patients with BCNS (6). Differences in type and onset of symptoms in BCNS patients from different ethnic populations clearly demonstrate the participation of other factor(s) besides PTCH1 in BCNS phenotypes. Data from BCNS patients with BCC support Knudson's two-hit hypothesis $(22,23)$, a theory of oncogenesis stipulating that normal cells require two mutagenic hits (two distinct episodes of DNA damage) to generate cancer. In BCNS, various benign and malignant tumors, such as BCC, KCOT, medulloblastoma and ovarian fibromas, exhibit loss of heterozygosity (LOH) $(3,24)$. Pan et al reported that not all KCOTs in BCNS-derived as well as sporadic KCOT had two PTCH1 mutations (25). Some KCOTs, as well as the sKCOT1 cell line, carry loss of function mutations in both $\mathrm{PTCH} 1$ alleles. Previously, we reported PTCHI mutations in both alleles of a BCNS-derived ovarian fibroma case (20). Therefore, loss of PTCHI functions itself does not confirm tumor malignancy. Further genome-wide analysis of 

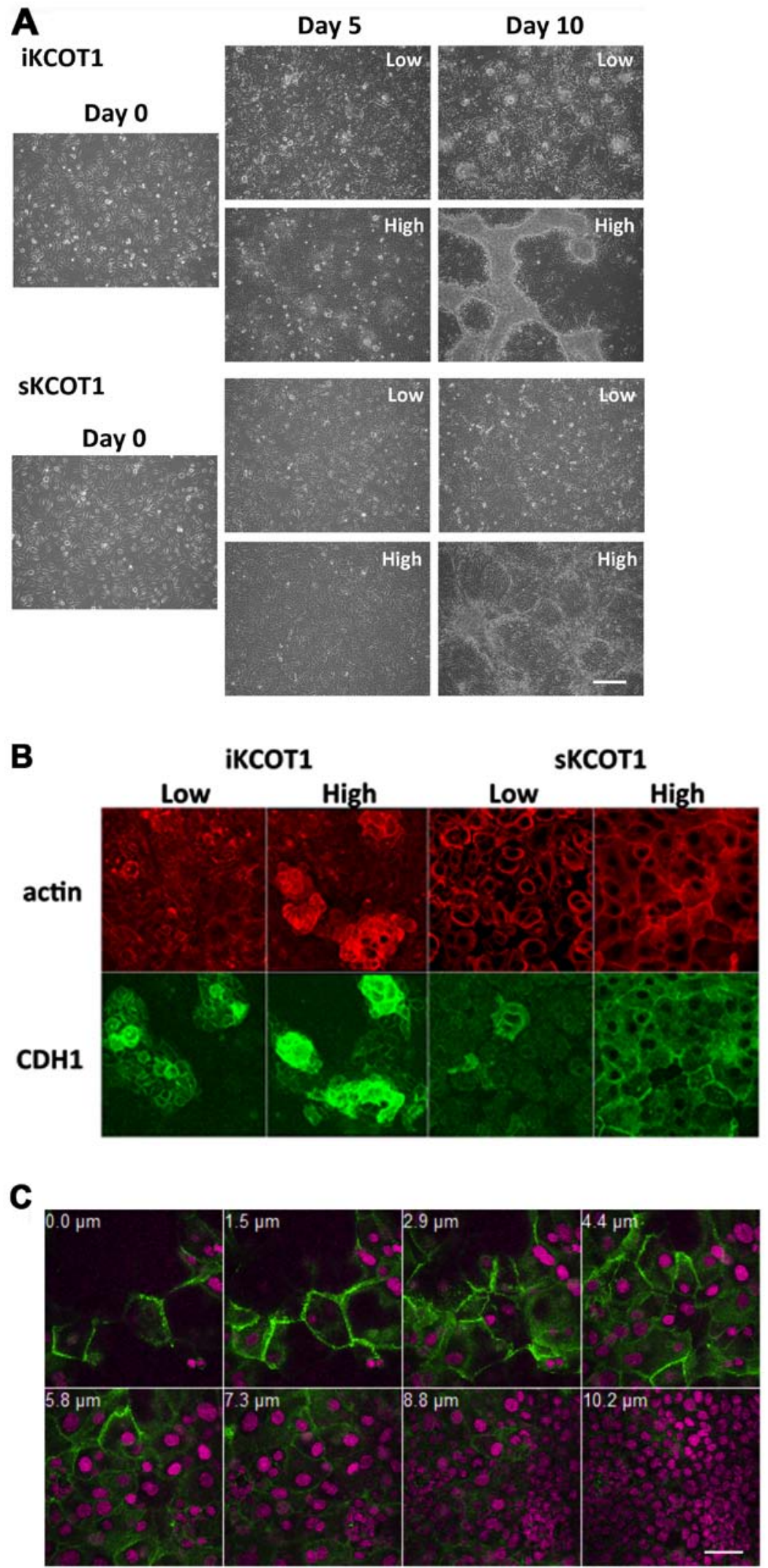

Figure 2. In vitro differentiation of KCOT cell lines by calcium. (A) Morphology of KCOT cells in media containing different calcium concentrations Cells were cultured in low calcium medium $(0.65 \mathrm{mM})$ (low) or high calcium medium $(1.23 \mathrm{mM})$ (high) for 10 days, in normal culture dishes. Bar indicates $200 \mu \mathrm{m}$. (B) Immunostaining of KCOT cells cultured in dishes with high or low calcium medium for 10 days, stained for CDH1 (green) and with actin (red). (C) Sequential images of sKCOT1 cells, stained for CDH1 (green) and DAPI (magenta). Cells on the surface showed parakeratosis, characteristic of KCOTs. Bar indicates $20 \mu \mathrm{m}$.

KCOTs will elucidate additional mutations or alterations that are involved in the malignancy of the tumor. Retinoblastoma is a malignant eye cancer that is common in childhood. It is known that loss of function of both copies of the tumor 

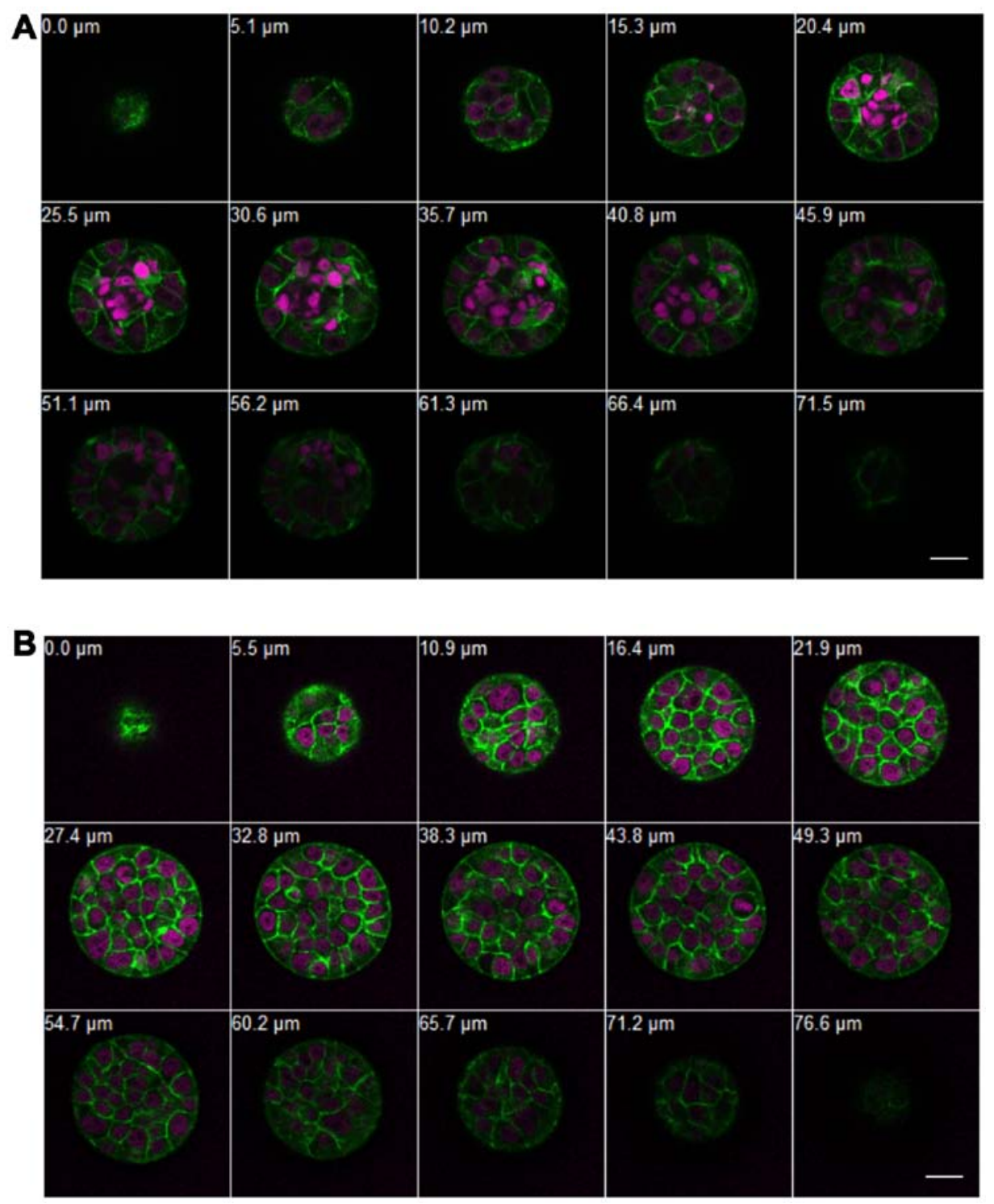

Figure 3. 3-D culture of KCOT cell lines. Sequential images of KCOT cells cultured in a 3-D life PVA Hydrogel for 12 days stained with rhodamine phalloidin (green) and DAPI (magenta). (A) sKCOT1 cells in low calcium medium. (B) iKCOT1 cells in low calcium medium. Bar indicates $20 \mu \mathrm{m}$.

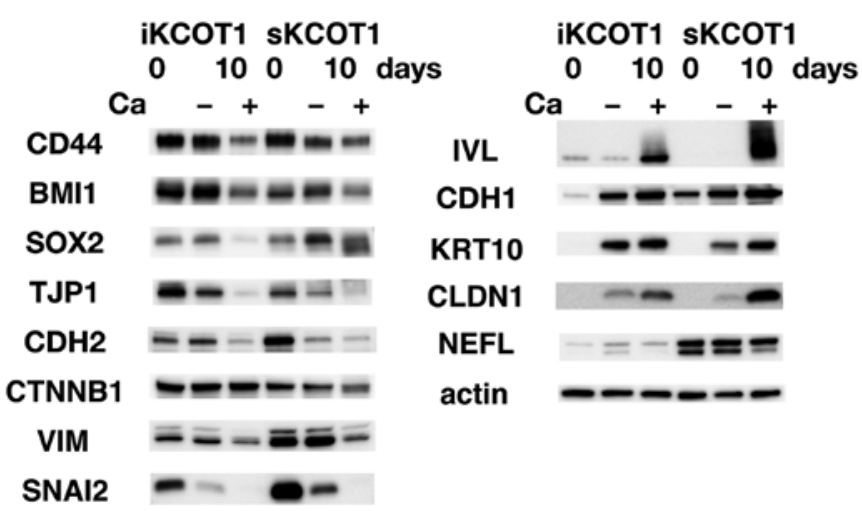

Figure 4. Protein expression in KCOT cell lines. Western blot analysis of KCOT cells grown in low $(0.65 \mathrm{mM})$ or high $(1.23 \mathrm{mM})$ calcium medium (indicating or + respectively) for 10 days. Stem cell markers, CD44, SOX2 and BMI1, were expressed; however, their levels were downregulated by the presence of calcium. Protein levels of mesenchymal-associated proteins, such as $\mathrm{CDH} 2$, VIM, CTNNB1 and SNAI2 were downregulated, whereas epithelial-associated proteins, such as $\mathrm{CDH} 1$ and CLDN1, were upregulated by the high calcium medium in both KCOT cell lines. Keratinocyte differentiation markers IVL and KRT10 were dramatically induced by the high calcium medium. suppressor $R B 1$ gene induces retinoblastoma. However, why depletion of the $R B I$ gene does not induce other types of cancers is not well understood. Xu et al showed that only cone precursor cells are sensitive to the $R b$ defect in mice and that a cell type-specific gene network is critical for the formation of a tumor (26). In BCNS patients or $\mathrm{Ptch}^{+/-}$mice, PTCH1 function is halved throughout the body; however, only specific cells or tissues show tumor formation. These data indicated that in addition to the depletion of $P T C H 1$ function, cell typespecific gene networks may specify the sensitivity of cells and the development of tumors. Next-generation sequencing will elucidate the genes involved in tumor formation and gene expression networks that are essential for cell type restricted tumor formation.

KCOT cells differentiate in response to high calcium conditions. KCOT cell characteristics were examined by immunofluorescence and western blot analyses (Figs. 1B and 4). Both cell lines expressed stem cell markers, such as CD44, BMI1 and SOX2, and mesenchymal cell markers, including 
CDH2, VIM and SNAI2. However, levels of these proteins were decreased under high calcium conditions. Conversely, levels of epithelial cell markers, CDH1 and CLDN1, and also the keratinocyte markers, KRT10 and IVL were dramatically increased (Fig. 4). These data suggested that both cell lines have a mesenchymal stem cell-like character, and high calcium conditions induced differentiation of epithelial characteristics. Grachtchouk et al showed that disrupting normal Hh signaling at the epithelial cell rests of Malassez (ERM) induced KCOTs (14). Gao et al induced KCOTs from ERM by disrupting TGF- $\beta$ signaling (27). ERM are odontogenic epithelial cells in the periodontal ligaments and are normally quiescent and predicted to work during periodontal regeneration $(28,29)$. ERM are known to express several proteins that are usually associated with mesenchymal cells (28). Therefore, ERM are a candidate for the origin of KCOTs. Recent analysis reveals that ERM have stem cell-like properties with capacities to undergo epithelial-mesenchymal transition (EMT) and to differentiate into mesenchymal lineages (29). Cells in which proliferation or differentiation activity is activated or repressed by abnormal Hh or TGF- $\beta$ signaling in ERM may be an origin of KCOTs. Therefore, ERM-derived KCOT cells may also have stem cell and mesenchymal properties. Alternatively, KCOT may be a result of EMT of ERM cells and a high calcium concentration induces reverse transition of KCOT cells from mesenchymal cells to epithelial-like cells. One interesting feature of these cell lines was the expression of NEFL, which is characteristic for neuronal cells (Fig. 4). This may be due to the mesenchymal stem cell-like characteristics of these cell lines. Recently, genome-wide expression analysis of fresh Ameloblastoma and KCOT samples were carried out, the KCOTs are distinguished by elevated levels of expression of epithelial differentiation markers, and stem cell marker SOX2 (30). These expression profiles partially support our KCOT cell lines as retaining original KCOT characteristics.

In conclusion, we have established immortalized KCOT cell lines, and have developed an in vitro culture model of KCOT. Our experimental models may facilitate further studies to understand the genesis of syndromic and sporadic KCOTs and provide a unique resource to elucidate unsolved questions.

\section{Acknowledgements}

We thank all patients, their families and collaborating doctors for participating in this study. We also thank the Joint-Use Research Facilities at Hyogo College of Medicine. This study was partly supported by Grants-in-Aid for Research on Intractable Disease from the Ministry of Health, Labour and Welfare, Japan (no. H22-120 to K.N.), and by JSPS KAKENHI (grant nos. 24592854 and 15K11098 to Y.N. and nos. 25463123 and $16 \mathrm{~K} 11737$ to K.N.), also supported by Grant-in Aid for Researchers, Hyogo College of Medicine (to Y.N.) 2015.

\section{References}

1. Morgan TA, Burton CC and Qian F: A retrospective review of treatment of the odontogenic keratocyst. J Oral Maxillofac Surg 63: 635-639, 2005.

2. Philipsen HP and Reichart PA: Classification of odontogenic tumours. A historical review. J Oral Pathol Med 35: 525-529, 2006.
3. Shear M: The aggressive nature of the odontogenic keratocyst: Is it a benign cystic neoplasm? Part 1 . Clinical and early experimental evidence of aggressive behaviour. Oral Oncol 38: 219-226, 2002.

4. González-Alva P, Tanaka A, Oku Y, Yoshizawa D, Itoh S, Sakashita H, Ide F, Tajima Y and Kusama K: Keratocystic odontogenic tumor: A retrospective study of 183 cases. J Oral Sci 50: 205-212, 2008

5. Cohen MM Jr: Nevoid basal cell carcinoma syndrome: Molecular biology and new hypotheses. Int J Oral Maxillofac Surg 28: 216-223, 1999.

6. Kimonis VE, Goldstein AM, Pastakia B, Yang ML, Kase R, DiGiovanna JJ, Bale AE and Bale SJ: Clinical manifestations in 105 persons with nevoid basal cell carcinoma syndrome. Am J Med Genet 69: 299-308, 1997.

7. Johnson RL, Rothman AL, Xie J, Goodrich LV, Bare JW, Bonifas JM, Quinn AG, Myers RM, Cox DR, Epstein EH Jr, et al: Human homolog of patched, a candidate gene for the basal cell nevus syndrome. Science 272: 1668-1671, 1996.

8. Ingham PW, Nakano Y and Seger C: Mechanisms and functions of Hedgehog signalling across the metazoa. Nat Rev Genet 12: 393-406, 2011.

9. Wicking C, Shanley S, Smyth I, Gillies S, Negus K, Graham S, Suthers G, Haites N, Edwards M, Wainwright B, et al: Most germ-line mutations in the nevoid basal cell carcinoma syndrome lead to a premature termination of the PATCHED protein, and no genotype-phenotype correlations are evident. Am J Hum Genet 60: 21-26, 1997 .

10. Fujii M, Noguchi K, Urade M, Muraki Y, Moridera K, Kishimoto $\mathrm{H}$, Hashimoto-Tamaoki $\mathrm{T}$ and Nakano Y: Novel PTCH1 mutations in Japanese Nevoid basal cell carcinoma syndrome patients: Two familial and three sporadic cases including the first Japanese patient with medulloblastoma. J Hum Genet 56: 277-283, 2011.

11. Qu J, Yu F, Hong Y, Guo Y, Sun L, Li X, Zhang J, Zhang H, Shi R, Chen F, et al: Underestimated PTCH1 mutation rate in sporadic keratocystic odontogenic tumors. Oral Oncol 51: 40-45, 2015.

12. Kimi K, Ohki K, Kumamoto H, Kondo M, Taniguchi Y, Tanigami A and Ooya K: Immunohistochemical and genetic analysis of mandibular cysts in heterozygous ptc knockout mice. J Oral Pathol Med 32: 108-113, 2003.

13. Svärd J, Heby-Henricson K, Persson-Lek M, Rozell B, Lauth M, Bergström A, Ericson J, Toftgård R and Teglund S: Genetic elimination of suppressor of fused reveals an essential repressor function in the mammalian hedgehog signaling pathway. Dev Cell 10: 187-197, 2006

14. Grachtchouk M, Liu J, Wang A, Wei L, Bichakjian CK, Garlick J, Paulino AF, Giordano T and Dlugosz AA: Odontogenic keratocysts arise from quiescent epithelial rests and are associated with deregulated hedgehog signaling in mice and humans. Am J Pathol 169: 806-814, 2006.

15. Ren C, Amm HM, DeVilliers P, Wu Y, Deatherage JR, Liu Z and MacDougall M: Targeting the sonic hedgehog pathway in keratocystic odontogenic tumor. J Biol Chem 287: 27117-27125, 2012.

16. Liu X, Ory V, Chapman S, Yuan H, Albanese C, Kallakury B, Timofeeva OA, Nealon C, Dakic A, Simic V, et al: ROCK inhibitor and feeder cells induce the conditional reprogramming of epithelial cells. Am J Pathol 180: 599-607, 2012.

17. Sasaki R, Narisawa-Saito M, Yugawa T, Fujita M, Tashiro H, Katabuchi $\mathrm{H}$ and Kiyono T: Oncogenic transformation of human ovarian surface epithelial cells with defined cellular oncogenes. Carcinogenesis 30: 423-431, 2009.

18. Yamamura M, Noguchi K, Nakano Y, Segawa E, Zushi Y, Takaoka K, Kishimoto H, Hashimoto-Tamaoki T and Urade M: Functional analysis of Zyxin in cell migration and invasive potential of oral squamous cell carcinoma cells. Int J Oncol 42: 873-880, 2013.

19. Athar M, Li C, Kim AL, Spiegelman VS and Bickers DR: Sonic hedgehog signaling in Basal cell nevus syndrome. Cancer Res 74: 4967-4975, 2014.

20. Jimbo T, Masumoto K, Urita Y, Takayasu H, Shinkai T, Uesugi T, Gotoh C, Sakamoto N, Sasaki T, Oto T, et al: Nevoid basal cell carcinoma syndrome with a unilateral giant ovarian fibroma in a Japanese 6-year-old girl. Eur J Pediatr 173: 667-670, 2014.

21. Takahashi C, Kanazawa N, Yoshikawa Y, Yoshikawa R, Saitoh Y, Chiyo H, Tanizawa T, Hashimoto-Tamaoki T and Nakano Y: Germline PTCH1 mutations in Japanese basal cell nevus syndrome patients. J Hum Genet 54: 403-408, 2009. 
22. Knudson AG Jr: Mutation and cancer: Statistical study of retinoblastoma. Proc Natl Acad Sci USA 68: 820-823, 1971.

23. Levanat S, Gorlin RJ, Fallet S, Johnson DR, Fantasia JE and Bale AE: A two-hit model for developmental defects in Gorlin syndrome. Nat Genet 12: 85-87, 1996.

24. Barreto DC, Gomez RS, Bale AE, Boson WL and De Marco L: PTCH gene mutations in odontogenic keratocysts. J Dent Res 79: $1418-1422,2000$

25. Pan S, Dong Q, Sun LS, Li TJ.: Mechanisms of inactivation of PTCH1 gene in nevoid basal cell carcinoma syndrome: modification of the two-hit hypothesis. Clin Cancer Res 16: 442-450, 2010.

26. Xu XL, Singh HP, Wang L, Qi DL, Poulos BK, Abramson DH, Jhanwar SC and Cobrinik D: Rb suppresses human coneprecursor-derived retinoblastoma tumours. Nature 514: 385-388, 2014.
27. Gao Y, Yang G, Weng T, Du J, Wang X, Zhou J, Wang S and Yang X: Disruption of Smad4 in odontoblasts causes multiple keratocystic odontogenic tumors and tooth malformation in mice. Mol Cell Biol 29: 5941-5951, 2009.

28. Rincon JC, Young WG and Bartold PM: The epithelial cell rests of Malassez - a role in periodontal regeneration? J Periodontal Res 41: 245-252, 2006.

29. Xiong J, Mrozik K, Gronthos S and Bartold PM: Epithelial cell rests of Malassez contain unique stem cell populations capable of undergoing epithelial-mesenchymal transition. Stem Cells Dev 21: 2012-2025, 2012.

30. Heikinheimo K, Kurppa KJ, Laiho A, Peltonen S, Berdal A, Bouattour A, Ruhin B, Catón J, Thesleff I, Leivo I, et al: Early dental epithelial transcription factors distinguish ameloblastoma from keratocystic odontogenic tumor. J Dent Res 94: 101-111, 2015. 